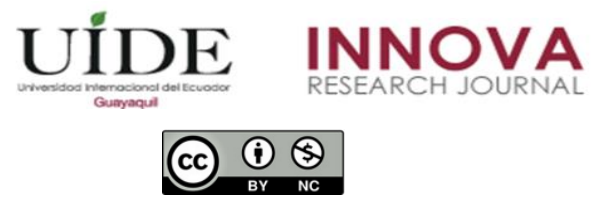

INNOVA Research Journal, ISSN 2477-9024

(Septiembre-Diciembre 2020). Vol. 5, No.3 pp. 62-77

DOI: https://doi.org/10.33890/innova.v5.n3.2020.1362

URL: http://revistas.uide.edu.ec/index.php/innova/index

Correo: innova@uide.edu.ec

\title{
Balanced Scorecard como Herramienta de Gestión y mejora en los Emprendimientos
}

\section{Balanced Scorecard as a Management and Improvement tool for Entrepreneurship}

\author{
Alexis Fernando Bolaños Jijón \\ https://orcid.org/0000-0002-2141-9682 \\ Universidad de Guayaquil, Ecuador \\ Julio César Méndez Bravo \\ https://orcid.org/0000-0003-0312-1775 \\ Universidad de Guayaquil, Ecuador \\ Universidad Internacional del Ecuador, Ecuador \\ Manuel Méndez Bravo \\ https://orcid.org/0000-0003-4103-8198 \\ Unidad Ecuativa Olida Dueñas Moreira, Ecuador
}

Autor para correspondencia: alexis.bolanosj@ug.edu.ec; julio.mendezb@ug.edu.ec; jumendezbr@uide.edu.ec; ing.com.manuelmendez@hotmail.com

Fecha de recepción: 02 de marzo del 2020 - Fecha de aceptación: 27 de junio del 2020

\section{Resumen}

El presente artículo realiza un análisis bibliográfico del Balanced Scorecard (BSC) en empresas para dar a conocer las oportunidades que presenta la implementación de esta herramienta. Se emplearon métodos de exploración teórica, análisis y síntesis de documentos tales como revistas y libros de varios autores acerca de los conceptos generales del Balanced Scorecard relacionados con emprendimientos los mismos que fueron presentados en tablas. El objetivo del presente documento es realizar un análisis del Balanced Scorecard herramienta de desarrollo y sostenibilidad para emprendimientos. En la actualidad, los emprendimientos están en auge, debido que brindan una oportunidad de generar ingresos, dinamizar la economía e introducir nuevos productos al mercado y, de esta manera, ofrecer variadas opciones de elección para el consumidor. Este estudio reveló que el Balanced Scorecard puede permitir al emprendedor, a través de su estructura de perspectivas, visualizar y hacer seguimiento a la estrategia definida, mejorar sus procesos internos, conocer las necesidades de sus clientes y obtener mayores ganancias en un mediano y largo plazo. El empleo de esta herramienta por los emprendimientos, incidirá decisivamente en el cumplimiento de los objetivos de estas organizaciones.

Palabras claves: emprendimiento; balanced scorecard; indicadores de gestión; desempeño; mejora 


\begin{abstract}
This article performs a bibliographic analysis of the Balanced Scorecard (BSC) in companies to publicize the opportunities presented by the implementation of this tool. Methods of theoretical exploration, analysis and synthesis of documents such as magazines and books by various authors about the general concepts of the Balanced Scorecard related to ventures were used, which were presented in tables. The objective of this document is to carry out an analysis of the Balanced Scorecard development and sustainability tool for enterprises. Currently, the ventures are booming, because they provide an opportunity to generate income, boost the economy and introduce new products to the market and, in this way, offer various options of choice for the consumer. This study revealed that the Balanced Scorecard can allow the entrepreneur, through its perspective structure, to visualize and monitor the defined strategy, improve its internal processes, meet the needs of its clients and obtain greater profits in the medium and long term. . The use of this tool by the enterprises will have a decisive impact on the fulfillment of the objectives of these organizations.
\end{abstract}

Key words: entrepreneurship; balanced scorecard; management indicators; performance; improvement

\title{
Introducción
}

Balanced Scorecard (BSC) o Cuadro de Mando Integral, es una herramienta gerencial que establece la implementación de estrategias que permitan el cumplimiento de la misión y estrategia de las organizaciones, también define sus objetivos y evalúa su cumplimiento, relacionándolos de forma definitiva y permanente con el control de su ejecución (Montoya, 2011) (Baigen, Guillén , \& Peinado, 2008).

El Balanced Scorecard traduce el lenguaje estratégico a uno operativo a través de la gestión y alineación de los objetivos, empleando métodos que facilitan la comunicación de la estrategia traducida a todos los niveles de la organización, lo cual permitirá una acción más efectiva de la organización (Gómez, 2018).

Los creadores del BSC, (Kaplan \& Norton , 1996) establecieron que su principal objetivo es convertir la estrategia de la empresa en acción y resultados mediante el desarrollo de sus cuatro perspectivas: financiera, clientes, procesos internos y aprendizaje. Para (Alvarez, Chávez, \& Moreno, 2005) el BSC es un proceso descendente que busca convertir la misión y estrategia de la organización en objetivos por para inducir la acción empresarial oportuna y relevante.

Una de las principales novedades que ofrece el BSC es que otorga la posibilidad de visualizar el desempeño de la organización en el tiempo, vinculando las perspectivas con el valor que genera. De esta manera los directivos pueden observar la evolución del rendimiento de la organización y estimar el futuro (Suárez \& González, 2017).

Por consiguiente, el BSC se puede considerar una herramienta esencial en el diseño de planificaciones estratégicas al permitir la comunicación y descripción de las estrategias de forma clara y coherente. Los directivos de las organizaciones son los que tienen que tomar decisiones estratégicas, los cuales se fundamentan en la determinación de políticas y objetivos y en la provisión de los recursos necesarios para la operación (Gómez, 2018). 
BSC trata de identificar líneas de acción que permitan a la organización lograr el despliegue de la estrategia (Suárez \& González, 2017). Lo más importante en la comunicación de la estrategia es procurar que todos la entiendan. El método que emplea el BSC para alinear la estrategia con los objetivos de la organización y con las perspectivas es a través del mapa estratégico (Peinado, Cerecedo, \& Jaramillo, 2016).

El BSC, respecto a la planeación estratégica, es un modelo que traduce la visión de la organización en objetivos o factores clave de éxito y que a su vez, se convierten en metas concretas y claras cuya consecución se mide a través de indicadores de desempeño de la empresa para aplicar la mejora continua en base a las cuatro perspectivas, las cuales permitirán llevar un control de la empresa facilitando la compresión del cuánto y cómo los empleados impactan en el desempeño y resultados de la empresa.

Entre los principales beneficios que provee el BSC, tenemos: provee información de las variables financieras y no financieras de la organización, alinea la estrategia a los objetivos y cuatro perspectivas, comunica la estrategia a toda la estructura organizacional, tanto horizontal como vertical, permite la revisión de la estrategia organizacional para su pronta adaptación de acuerdo a los cambios del entorno, facilita la formación de manera específica, promueve la mejora continua (Amat, Banchieri, \& Campa-Planas, 2016).

Una de las principales ventajas de esta herramienta es que puede ser aplicada a todo tipo de organizaciones: micros, pymes, grandes empresas, entidades públicas, organizaciones sin fines de lucro, fundaciones, ONGs, y demás (Mc Coy, Martinez, \& Aguiar, 2019) (Suárez \& González, 2017).

El emprendedor es aquel cuyo accionar está influenciado por factores propios que, por los ajenos a la organización, sin embargo, se han determinado ciertamente cuales son dichos factores. Por tal razón, se considera de gran importancia el determinar dichas características internas que deberían poseer los emprendedores, las cuales influirán a quienes estén dispuestos a iniciar emprendimientos como su forma de generar desarrollo en su ciudad y país (Salvador, 2008).

Al analizar la historia de la humanidad, se debe considerar al emprendedor en distintos aspectos relevantes. En los últimos años el concepto de emprendimiento se ha vuelto imprescindible debido que surge de la necesidad de superar los problemas económicos (Maldonado, 2018). Para (Guzmán \& Trujillo, 2008) la economía busco relacionar al BSC con el desarrollo, pero desde un enfoque netamente financiero. Luego otras ciencias, como la psicología, sociología y antropología generaron aportes desde un enfoque social, lo que mejoro su comprensión y aporte.

Para (Holguín, 2013) la formación de emprendimientos demanda de nuevas y diversas metodologías que rompa los paradigmas de la pedagogía, donde se vincule al mundo real de las empresas, servicios y diversas instituciones de la sociedad, permitiendo fortalecer al individuo en sus habilidades intrapersonales y emprendedoras para establecer alianzas y redes con otros individuos, permitiéndoles ser conscientes de la realidad social y del mercado laboral, convirtiendo en un individuo responsable de sus acciones y decisiones. 
Debido que los emprendedores buscan crear valor para su empresa explotando las diversas oportunidades que le permitan incrementar sus ingresos generando ganancias a corto y largo plazo, la implementación de BSC en los emprendimientos permitirá un mejor manejo del mismo en función de los recursos materiales como humanos. Mediante la aplicación de esta herramienta estratégica, con ayuda de sus perspectivas en base a los puntos más relevantes, se podrán desarrollar indicadores que permitirán obtener resultados con el fin de optimizar cambios en todos los procesos de la empresa, que permita mostrar mejores resultados, enfocándose no solo en los aspectos personales y medioambientales, sino también, considerando las diferentes actitudes que influyen dentro del emprendimiento.

\section{Metodología}

La metodología es cualitativa de revisión bibliográfica. Se emplearon métodos de exploración teórica, análisis y síntesis de documentos tales como revistas de alto impacto y libros. Adicionalmente a los documentos referenciados se revisaron aproximadamente 80 artículos como documentos de consulta complementarios de la información desarrollada acerca de los conceptos generales del Balanced Scorecard, sus perspectivas y sus beneficios relacionados con emprendimientos.

\section{Resultados}

Para (Krauss, 2011) el emprendedor se considera al individuo que asume riesgos, y que no teme arriesgarse a cosas nuevas, por lo tanto, el emprendedor no tiene temor a independizarse y crear su propio negocio que permita generar nuevos plazos de trabajo. Por consiguiente, se puede definir al como persona visionaria, que posee iniciativa al momento de crear una empresa, es aquel que tiene la capacidad de observar y hallar problemas por lo cual toma decisiones sin importar los riesgos que se le presenten logrando alcanzar sus objetivos para así, generar ganancias.

Por lo tanto, un emprendedor no solo puede ser un gerente, sino también un colaborador, una persona que labora en la empresa. (Thompson, 2009) en su investigación mencionó que la intención emprendedora es un aspecto relevante de quienes desean estudiar una carrera empresarial o afín, sin embargo, no todos logran transformar en realidad sus aspiraciones.

Emprender es crear, planificar, actuar llevando a cabo su idea generando oportunidades y recursos que se necesitan, verificando si el espíritu emprendedor genera efectos positivos en una comunidad desarrollando una cultura de emprendimiento. Emprender es perseguir la oportunidad más allá de los recursos que se controlen en la actualidad, en la medida que el emprendedor no nace, sino que se hace (Ruiz, García, \& Delgado, 2014). Por lo cual el emprendimiento es un proceso de aprendizaje que a través de diferentes métodos permitirán a las personas crear o identificar oportunidades que lleven a la innovación.

Se define al emprendedor como aquella persona que organiza y dirige una empresa o una nueva actividad, toma riesgos para obtener mayor ganancia y ve al cambio como una nueva oportunidad de desarrollo económico en lugar de un problema que debe afrontar. Así también (Durán, Parra, \& Márceles, 2015) se refirieron a la actitud emprendedora como la disposición de 
un individuo para actuar frente a cualquier situación que se presente. La actitud emprendedora permite generar ideas innovadoras que pueden transformarse en proyectos y negocios que busca satisfacer una necesidad o solucionar una problemática existente, además de generar el crecimiento económico y desarrollo personal.

Otra definición de los emprendedores es que son las personas que esperan iniciar, no solo una carrera empresarial, sino aquella que tiene una idea, la plasma en un proyecto y la lleva a la ejecución, cuentan con una actitud emprendedora que les permite desarrollar nuevas ideas innovadoras y llevarlas a la realidad como soluciones a problemáticas, llegando así a la mejora continua en los proyectos planteados.

\section{BSC recomendable para los emprendedores}

El BSC se lo considera como un sistema de gestión estratégico y de medición de rendimiento que aparece apto para ser utilizado por todos los tipos y tamaños de negocios. La fortaleza del BSC, para la mayoría de las empresas, proviene de su capacidad innata de integrar medidas financieras y no financieras en conjunto, mediante la medición tanto estratégica como de desempeño del negocio a través de cuatro perspectivas interrelacionadas (Giannopoulos, Holt, Khansalar, \& Cleanthous, 2013).

El BSC va más allá de ser una simple herramienta, debido que permite a los diferentes niveles de la dirección estar informados de las múltiples estrategias que se llevan a cabo. En el caso de los emprendimientos, el uso de esta herramienta permite que se creen estrategias y se analicen los puntos críticos en base a los indicadores que se incluyen en la misma, además de sus diferentes perspectivas las cuales optimizarán una visión de los factores más relevantes.

Los emprendedores crean sus negocios para tener una fuente de ingresos, y esta herramienta permite evaluar su desempeño mediante las cuatro perspectivas: (a) financiera; (b) clientes; (c) procesos internos; y (d) aprendizaje, empleando indicadores, los cuales serán de vital ayuda para que se puedan cumplir los objetivos y mejorar los procesos, obteniendo mejores resultados y encaminando al negocio a un crecimiento a largo plazo.

\section{Perspectivas del BSC}

Lo que más caracteriza al BSC son sus perspectivas. El uso de la palabra perspectiva es intencionado, es un término genérico y que exclusivamente refleja el punto de vista con el que se identifica esta herramienta (Quintero-Beltrán \& Osorio-Morales, 2018). En la Tabla 1 se describen las cuatro perspectivas con sus respectivos objetivos en la organización.

\section{Balanced Scorecard}

Se ha realizado una amplia lectura de artículos de diferentes estudios realizados en diversos países, por los cuales se pudo determinar las diversas aplicaciones del BSC, por ejemplo (Vásquez \& Carrillo, 2010) investigaron como las instituciones de educación superior pueden apoyarse en el BSC mediante la creación de indicadores de gestión. Otro ejemplo es el estudio del BSC como herramienta para las empresas (Quintero-Beltrán \& Osorio-Morales, 2018). 
Tabla. 1

Análisis de las perspectivas.

\begin{tabular}{ll}
\hline Perspectiva & Objetivo \\
\hline Perspectiva financiera & $\begin{array}{l}\text { Describir los resultados tangibles, busca } \\
\text { incrementar los ingresos esperando obtener buenos } \\
\text { resultados. }\end{array}$ \\
Perpectiva cliente & $\begin{array}{l}\text { Reflejar el posicionamiento de la empresa en el } \\
\text { mercado mediante la fidelización y satisfacción del } \\
\text { cliente. }\end{array}$ \\
Perspectiva procesos internos & $\begin{array}{l}\text { Identificar los procesos internos que generan } \\
\text { mayor impacto en la satisfacción del cliente, de } \\
\text { innovación, de medio ambiente, creando } \\
\text { estrategias en los procesos de su estructura } \\
\text { organizacional. }\end{array}$ \\
Perspectiva aprendizaje y desarrollo & $\begin{array}{l}\text { Se identifican las competencias que debe } \\
\text { desarrollar el capital humano y así puedan generar } \\
\text { estrategias de motivación, capacitación, mejorar de } \\
\text { tecnologías etc. }\end{array}$ \\
\hline
\end{tabular}

Fuente: Elaboración propia

La evolución del BSC desde su desarrollo en 1992 por Norton y Kaplan ha sido relevante, inicio como una herramienta de control de gestión mediante la implementación de indicadores no financieros, los cuales permitían explicar los resultados financieros actuales y prever los futuros. Para (Amo, 2011) el BSC ofrece a las empresas una metodología que se adapta a las necesidades de la gestión de la organización así como a su cultura organizacional y cualificación de los directivos que posee.

Para (Alves , Ferraz, Chiari, Ofenhjem , \& Goncalves, 2014) el BSC se puede aplicar para diferentes requerimientos que van desde una herramienta de medición de desempleo hasta el desarrollo de un sistema integrado de estrategias. Actuando como foco en la visión y estrategia de la empresa mediante interconexiones que se generan en las cuatro perspectivas.

El desafío más grande que enfrenta una empresa es medir su rendimiento, el cual es un análisis clave a la hora de desarrollar sus planes estratégicos, apreciación de sus logros en función de los objetivos planteados, la evaluación de incentivos, entre otros. Para Kaplan (1983) citado por (Aguayo, 2004) las nuevas circunstancias forzaban a pasar del énfasis de las medidas financieras a corto plazo, a las no financieras del largo plazo, por ello el ámbito contable de gestión no solo debe de enfocarse en la parte financiera, sino también en indicadores de carácter no financiero, ya que las consideraciones más importantes de éxito se desarrollan en el largo plazo y no en el corto plazo.

Para (Morales \& Pinilla, 2007) el BSC es un modelo de gestión basado en estrategias, el cual busca transformarla en acción, orientándola al éxito en ambientes de alta incertidumbre. Integrando los objetivos estratégicos de las perspectivas al modelo de gestión, los cuales se relacionan entre sí, desarrollando la causa-efecto. Además de los objetivos estratégicos, el BSC cuenta con indicadores, alineación y aprendizaje estratégico con los que monitorea el desempeño 
de la organización en base a la estrategia implementada. Por lo tanto, es una herramienta diagnostica de la estrategia sobre la base de los objetivos, perspectivas y el causa-efecto.

Según (Gómez, 2018) el BSC se lo puede definir como una metodología que ayuda a transformas las estrategias de las empresas en objetivos operativos medibles y que se pueden relacionarse entre sí, alineando estratégicamente el comportamiento de los colaboradores claves de la empresa así como el de sus recursos. El BSC se ha convertido en una herramienta útil y accesible no solo para las grandes empresas sino también para las pequeñas y medianas organizaciones teniendo en cuenta a donde se quiere llegar. En la Tabla 2 se detallan algunos artículos referentes al Balanced Scorecard, sus objetivos y conclusiones.

Para (Méndez, Ayala, \& Palacios, 2019) la importancia del BSC en las empresas familiares se determina dado que este tipo de empresas tiende a fracasar antes de cumplir los primeros cinco años, sin embargo, el BSC como sistema de gestión identifica los problemas y ayuda a mejorarlos, permitiendo sus subsistencia en los mercados.

La aplicación del BSC es tan versátil que se puede aplicar en diferentes tipos de organizaciones, (Vásquez \& Carrillo, 2010) desarrollaron su investigación en instituciones de educación superior con el objetivo de plantear la construcción de indicadores de gestión que les permita realizar el seguimiento, control y evaluar los resultados de los planes planteados, llegando a la conclusión que el BSC puede complementar las mediciones existentes, al incorporarles valor que reflejen la complejidad del sistema.

(Alves , Ferraz , Chiari, Ofenhjem , \& Goncalves, 2014) analizaron el desempeño de la planificación estratégica y de la formación de capital humano a largo plazo, desde la perspectiva del aprendizaje y el crecimiento, concluyendo que la creación de valor de las organizaciones se puede relacionar con la necesidad de invertir en la cultura y el aprendizaje continuo y en la gestión del conocimiento.

(Quintero-Beltrán \& Osorio-Morales, 2018) analizaron la pertinencia del BSC en empresas en crisis mediante la metodología de la exploración documental, donde determinaron que el BSC es una herramienta que no es un lujo sino inclusive llega a ser una necesidad para cualquier empresa. Dado que se creó con la intención de ser una herramienta útil y de ayuda para la prosperidad y sostenibilidad de una empresa.

(Muñoz, 2009) propuso el diseño y aplicación del BSC en unidades de información mediante un proceso de pasos específicos, al término de su estudio estableció al BSC como un instrumento necesario para el empleo de sistemas de control estratégico de gestión al relacionar la causalidad de sus resultados con los objetivos estratégicos establecidos.

(Aguayo, 2004) confronto observaciones de la realidad y marcha de la organización, con los respectivos propósitos, objetivos y estrategia establecida en ella, concluyendo que el BSC es más que una herramienta de control de gestión tradicional, ya que aporto como instrumento de comunicación estratégico en la organización. 
(Niven, 2003) desarrollo e implemento el BSC en una organización, y lo consideró como un componente global de los sistemas de gestión al aplicarlo en cascada sobre todos los niveles organizativos, vinculando a la estrategia el presupuesto y los incentivos.

(Amo, 2011) implemento un modelo de gestión con el BSC para permitir alinear el comportamiento del personal de la organización con la estrategia de la empresa. Al término de su trabajo estableció que el BSC ayuda a la empresa a transformar su estrategia en objetivos operativos y relacionados entre sí.

(Pérez \& García , 2014) evaluaron el desempeño de la organización y transformaron el sistema de gestión para implementar estrategias empresariales con lo cual determinaron que la construcción de un BSC en una empresa como instrumento de control de gestión logra la alineación estratégica.

Como se puede apreciar, el BSC es una herramienta versátil adaptable a las necesidades de las organizaciones, sin embargo, en todas cumple un mismo objetivo fundamental, el cual es transformar en acción la misión y visión de la organización mediante el cumplimiento de sus objetivos estratégicos y bajo el monitoreo de sus indicadores.

\section{Emprendimiento}

Como mencionó (Juliá, 2012) en su artículo, el termino emprendimiento deriva de la voz castellana emprender que proviene del latín colligere, el cual describe a un individuo que organiza y opera en una empresa asumiendo riesgo financiero para hacerlo. La persona emprendedora empieza a desarrollar su idea innovadora por medio de una necesidad la cual será la generadora de una oportunidad de negocio.

El emprendimiento no es más que una búsqueda de soluciones de una problemática basada en una necesidad. También podría llegar a crear su propia necesidad, es decir desarrollar una idea que haga que las personas se sientan dependientes de ella. La idea debe basarse en el desarrollo creativo e innovador, además de tener en cuenta un sinnúmero de factores que pueden intervenir. Con la implementación del BSC se obtendría una mejor visión hacia el pleno cumplimento de los objetivos con el fin de identificar los puntos claves que conlleven al éxito.

Un emprendedor debe ser un líder por excelencia, para (Méndez \& Méndez, 2016) la relación existente entre la personalidad del líder y su influencia en el estilo del liderazgo de quien guía y toma las decisiones en la organización son relevantes al ser el encargado del correcto funcionamiento de la organización y del cumplimiento de los objetivos planteados.

Para (Holguín, 2013) existe un fomento sobre la cultura emprendedora, la cual incentiva la innovación, permitiendo el crecimiento y posicionamiento de nuevos proyectos de emprendimiento en los mercados competitivos. (Juliá, 2012) también menciono que los emprendimientos deben contar con un componente innovador, debido a que estos son una función previa a la innovación, ya que de estos depende muchas veces el crecimiento económico. Se puede establecer que no solo se trata de cubrir una necesidad o de crear algo nuevo, el desarrollo de la idea debe generar beneficios sociales y económicos. 
(Nuñez \& Nuñez, 2016) consideran que pensar creativamente es un aspecto extraordinario de un individuo al dar una respuesta no conocida a un problema existente. El factor clave está en cómo se implementará y ejecutará esa solución, en la viabilidad, porque no toda alternativa a solución es la correcta. Esta debe cumplir con ciertos puntos clave que conlleven al éxito. Uno de los puntos clave sería identificar el objetivo, que es lo que se quiere lograr con la idea. Lo que permitirá obtener una mejor visión de los factores más importantes que se encontraran involucrados en la ejecución.

Los emprendimientos son una gran oportunidad de obtener ingresos, por consiguiente, la persona que vaya a realizar un emprendimiento debe tener conocimientos pertinentes en creación de estrategias y una considerable creatividad para llevar más allá su idea. En la actualidad existen muchas herramientas que pueden ayudar a mejorar procesos, en este caso aplicando la herramienta del BSC abrirá la puerta a la creación de estrategias basadas en sus perspectivas. Además de proporcionarnos una correcta visión del proyecto, sin olvidar que su estructura facilitará que todos los interesados y miembros comprendan y estén informados de igual manera de los objetivos y proceso.

(Guzmán \& Trujillo, 2008) establecieron el interés de las escuelas de negocios mediante la relación de la creación y dirección de empresas como razones principales, indicando que el objetivo del emprendimiento social es la solución a problemas sociales. Posteriormente (Landsdale, Abad, \& Vera, 2012) realizaron una investigación con el objetivo de demostrar que Ecuador se encontraba en un momento oportuno de implementación de estrategias para fomentar el desarrollo de emprendimientos, en la cual establecieron que el país se encuentra en el momento adecuado con un ecosistema dinámico que plantee la cultura de emprendimiento generadora del ansiado desarrollo económico. Esta apreciación es confirmada por Sepúlveda, que insiste en la importancia de promover una cultura del emprendimiento, el cual incide significativamente en el crecimiento de los países (Sepúlveda \& Reina, 2016)

(Hidalgo, 2013) desarrollo su investigación en instituciones superiores donde sustento la importancia de la formación profesional en el emprendimiento como una respuesta a las necesidades en el campo del conocimiento y práctica empresarial. Concluyendo que la formación en emprendimiento ofrece a los graduados una preparación real y capacidad de respuesta a las necesidades de la sociedad.

De la misma forma (Holguín, 2013) desarrollo su investigación en universidades de Medellín, buscando fortalecer los procesos y modelos de emprendimiento. Llegando a concluir que el modelo educativo por competencias fomenta de mejor manera los procesos formativos desarrollando la disciplina y el trabajo académico.

(Nuñez \& Nuñez, 2016) realizaron una revisión bibliográfica del emprendimiento en el contexto de educación escolar donde cualquier individuo puede generar una intención de emprender. Indicaron que la búsqueda de indicadores puede anticipar la decisión futura de emprender y determinar describir de mejora manera los aspectos del comportamiento del emprenderlo. 
(Villaroel, Cabrales, Fernández , \& Godoy , 2017) evaluaron los factores de innovación y emprendimiento mediante un análisis estadístico sobre la producción, estableciendo que las capacidades de innovación y emprendimiento son determinadas para mantener incrementos sostenidos de la actividad regional, y la competitividad de la región se puede mejorar mediante la implementación de políticas públicas adecuadamente diseñadas.

(Sánchez, Ward, Hernández, \& Florez, 2017) realizaron su investigación en el área de la educación, observando la incertidumbre sobre la empleabilidad de los individuos, considerando el autoempleo un rol relevante en las sociedades actuales, no solo por la necesidad, sino por los prospectos que genera la innovación y los resultados en mejora de la sociedad. Consideraron la educación emprendedora, indiscutiblemente, ha progresado desde que comenzó a conceptualizarse en escuelas de negocio como curso ha mediado del siglo pasado.

\section{BSC y Emprendimiento}

Para (Neftalí, 2018) el BSC puede considerarse la principal herramienta metodológica que traduce la estrategia de la organización en un conjunto de medidas e indicadores que proporcionan a la organización una estructura necesaria para un adecuado sistema de gestión y medición de resultados. Mientras que (Fernández, Mejía, Llerena, \& Albuja, 2017) los cambios constantes a nivel mundial debido a los desastres naturales y a los efectos de la globalización, han obligado a las organizaciones a generar nuevas opciones para ser competitivos y rentables con una menor inversión, para lo cual el BSC es la opción más viable, al lograr cambios planificados y organizados que se reflejan con una mejor participación en los mercados.

(Rigby \& Bilodeau, 2011) indicaron que diferentes estudios demostraron la popularidad del BSC, así como su implementación en diferentes tipos de organizaciones, entre las cuales se destacan los emprendimientos. Sánchez, Vélez y Araújo (2016) mencionan como los emprendedores desarrollan un modelo de negocio a partir de una idea. Lo cual puede facilitarse con la implementación del BSC.

(Cordero \& Zepeda, 2013) indicaron que la manera en que una organización se vuelve competitiva es con la implementación del BSC en el sentido estricto del desarrollo empresarial y de la mejora del desempeño. Complementado esta afirmación, (Bedoya, Toro, \& Arango, 2016) mencionaron que las actuales dinámicas de los entornos empresariales exigen un replanteamiento del actual rol del empresario tradicional. Un cambio transcendental se da con la adecuada implementación de una herramienta de gestión que permita mejorar la rentabilidad y la sostenibilidad de los emprendimientos. En la Tabla 2 se detallan algunos artículos referentes al Balanced Scorecard relacionados con el emprendimiento, sus objetivos y conclusiones.

Para (Sparano, 2014) el emprendedor nace con una idea de negocio al observar una necesidad que la transforma en oportunidad. Sin embargo, la transición de idea a realidad dependerá de que instrumento utilice para su realización, el BSC se considera como la herramienta de gestión que facilita su ejecución y monitoreo. 


\section{Tabla 2}

\section{Análisis de las Variables BSC y Emprendimiento}

\begin{tabular}{|c|c|c|}
\hline Autor & Objetivo de la Investigación & Conclusiones \\
\hline $\begin{array}{l}\text { (Fernández, } \\
\text { Mejía, } \\
\text { Llerena, \& } \\
\text { Albuja, } \\
\text { 2017) }\end{array}$ & $\begin{array}{l}\text { Diseñar un modelo de gestión empresarial } \\
\text { para la microempresa MEDSAKELL, } \\
\text { basado en la metodología BSC }\end{array}$ & $\begin{array}{l}\text { La planeación estratégica es fundamental } \\
\text { para el crecimiento de la organización y sus } \\
\text { objetivos estratégicos deben alinearse a la } \\
\text { misión y visión dela organización. }\end{array}$ \\
\hline $\begin{array}{l}\text { (Sánchez, } \\
\text { Vélez , \& } \\
\text { Araújo, } \\
\text { 2016) }\end{array}$ & $\begin{array}{l}\text { Determinar la complementabilidad del BSC y } \\
\text { la metodología CANVAS como herramientas } \\
\text { para los emprendedores. }\end{array}$ & $\begin{array}{l}\text { El BSC puede desarrollarse como una } \\
\text { herramienta complementaria a la } \\
\text { metodología CANVAS logrando poner en } \\
\text { marcha y desarrollar nuevos } \\
\text { emprendimientos. }\end{array}$ \\
\hline $\begin{array}{l}\text { (Montoya, } \\
\text { 2011) }\end{array}$ & $\begin{array}{l}\text { Reflexionar frente a los componentes que } \\
\text { ofrece el BSC a los procesos de la Gestión de } \\
\text { la empresa. }\end{array}$ & $\begin{array}{l}\text { El BSC es un sistema de gestión que } \\
\text { requiere de todos los miembros de la } \\
\text { organización compromiso y participación } \\
\text { para alcanzar los beneficios y el éxito } \\
\text { deseado. }\end{array}$ \\
\hline $\begin{array}{l}\text { (Morales \& } \\
\text { Pinilla, } \\
\text { 2007) }\end{array}$ & $\begin{array}{l}\text { Evaluar las capacidades de una organización a } \\
\text { la vez que se enrumba al éxito. }\end{array}$ & $\begin{array}{l}\text { El BSC permitió evaluar las capacidades de } \\
\text { la organización como fuertes o débiles en } \\
\text { relación a su desarrollo y articulación con la } \\
\text { misión y visión de la organización, así } \\
\text { como entre perspectivas. }\end{array}$ \\
\hline $\begin{array}{l}\text { (López, } \\
\text { Rojas, \& } \\
\text { Torres, } \\
\text { 2001) }\end{array}$ & $\begin{array}{l}\text { Establecer si el BSC busca actuar como } \\
\text { elemento integrador de los hechos actuales } \\
\text { con los futuros. }\end{array}$ & $\begin{array}{l}\text { El BSC debe estar según las condiciones } \\
\text { específicas de la organización que se } \\
\text { pretende controlar, por cuanto todo lo } \\
\text { planificado en la estrategia se alcanza. }\end{array}$ \\
\hline
\end{tabular}

Fuente: Elaboración propia

El interés de los emprendedores en conocer la implementación del BSC en los negocios surge mediante las oportunidades que éste origina, al saber que pueden generar mayores ganancias en el futuro, permitiendo tener una evaluación de los indicadores dentro de la empresa con respecto a las perspectivas, financiera, proceso interno, cliente y por último la perspectiva de desarrollo y aprendizaje, lo que conlleva a tener un mayor crecimiento a mediano y largo plazo a través de mejores resultados.

En la Figura 1 se puede apreciar la relación que se desarrolla entre el BSC y los emprendimientos, las dimensiones que tienden a influenciar en cada variable, así como el efecto que tiene el BSC en la sostenibilidad y desarrollo que permite generar en los emprendimientos. 


\section{Figura1}

Relación del Balanced Scorecard y el Emprendimiento

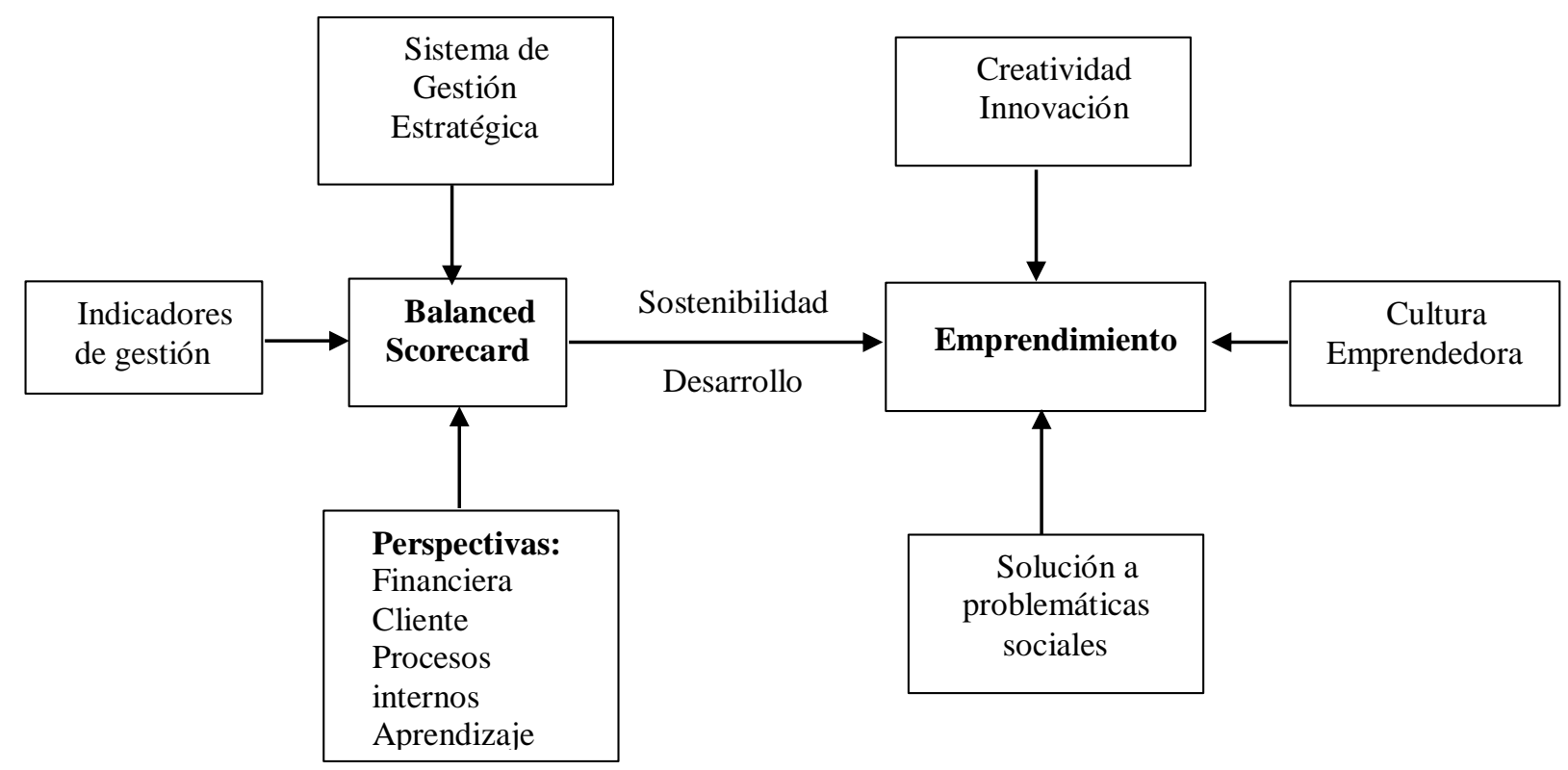

\section{Conclusión}

A lo largo del desarrollo, se encontró una breve descripción de los conceptos que abarca el BSC, conocido como el Balanced Scorecard, aplicado al emprendimiento. En las tablas 1 y 2 se puede observar las diferentes investigaciones de varios autores relacionados acerca del tema propuesto, donde se mencionó las perspectivas y los objetivos que son fundamentales para el desarrollo del trabajo en los emprendimientos y las oportunidades que surgen mediante la aplicación de una correcta metodología del BSC.

El interés de los emprendedores por tener un amplio conocimiento en la implementación del BSC en los emprendimientos, surge mediante diversas oportunidades que pueden generarse al conocer que el BSC permite tener mayor ganancia en el futuro el funcionamiento competitivo y exitoso, que pueda presentarse obteniendo mejores resultados en la correcta implementación del de esta metodología en los emprendimientos.

Para concluir, se podría decir que la implementación de la herramienta del BSC tienen un efecto positivo en los emprendimientos gracias a su estructura, el cual se enfoca en alcanzar el cumplimento de la misión. De igual manera, el BSC faculta la definición de objetivos alcanzables por medio de las perspectivas financiera, cliente, procesos internos y desarrollo y aprendizaje, los cuales permitirán el progreso óptimo del emprendimiento. Esta herramienta facilita la comunicación y ayuda a comprender de manera más sencilla los objetivos, logrando así obtener excelentes resultados presentando oportunidades y emprendimientos exitosos. 


\section{Bibliografía}

Aguayo, M. (2004). Las empresas y el Balanced Scorecard. Pharos, 11(2), 151-154. doi:https://www.redalyc.org/pdf/208/20811214.pdf

Alvarez, M., Chávez, M., \& Moreno, S. (2005). El Balanced Scorecard, una herramienta para la planeación estratégica. El Buzón de Pacioli, 1-16. doi:https://www.itson.mx/publicaciones/pacioli/Documents/no66/17ael_bsc_una_herramienta_para_la_planeacion_estrategicax.pdf

Alves , D., Ferraz , O., Chiari, E., Ofenhjem , A., \& Goncalves, W. (2014). La dinámica de la perspectiva aprendizaje y crecimiento del BSC. Invenio, 17(33), 87-102. doi:https://www.redalyc.org/pdf/877/87732404007.pdf

Amat, O., Banchieri, L., \& Campa-Planas, F. (2016). La implantación del cuadro de mando integral en el sector agroalimentario: el caso del Grupo Alimentario Guissona. Revista de la Facultad de Ciencias Económicas de la Universidad Militar Nueva Granada, 26-36. doi:http://dx.doi.org/10.18359/rfce.1619

Amo, F. (2011). El Cuadro de Mando Integral «Balanced Scorecard». Madrid: ESIC Editorial.

Baigen, A., Guillén , L., \& Peinado, J. (2008). Propuesta de Balance Scorecard para el centro de investigación y docencia económicas A.C. Investigación Administrativa(101), 67-76. Obtenido de https://www.redalyc.org/articulo.oa?id=456045196005

Bedoya, M., Toro, I., \& Arango, B. (2016). Emprendimiento Corporativo e Innovación: Una revisión y futuras líneas de investigación. Espacios, 38(17), 2-18. doi:https://www.revistaespacios.com/a17v38n17/a17v38n17p20.pdf

Cordero, I., \& Zepeda, L. (2013). Administración del desempeño organizacional a través del uso del Balanced Scorecard. Caso: All Natural \& Organic. Revista Iberoamericana de Contaduría, Economía y Administración, 2(4), 56-72. doi:10.23913/ricea.v2i4

Durán, S., Parra, M., \& Márceles, V. (2015). Potenciación de habilidades para el desarrollo de emprendedores exitosos en el contexto universitario. Opción, 31(77), 200-215. doi:https://www.redalyc.org/pdf/310/31041172012.pdf

Fernández, L., Mejía, S., Llerena, C., \& Albuja, J. (2017). Diseño de un modelo de gestión empresarial para la microempresa medsakell, basado en la metodología balanced scorecard, radicada en la zona 8, cantón Guayaquil. Espacios, 39(6), 1-11. doi:https://www.revistaespacios.com/a18v39n06/a18v39n06p36.pdf

Giannopoulos, G., Holt, A., Khansalar, E., \& Cleanthous, S. (2013). The Use of the Balanced Scorecard in Small Companies. International Journal of Business and Management, 8(14), 1-22. doi:10.5539/ijbm.v8n14p1

Gómez, O. (2018). Simulación del modelo de negocio de la ETB: de la contabilidad financiera a la simulación de la estrategia corporativa. Cuadernos de Contabilidad, 19(48). doi:https://doi.org/10.11144/Javeriana.cc19-48.smne

Guzmán , A., \& Trujillo, M. (2008). Emprendimiento social - revisión de literatura. Estudios Gerenciales, 24(109), 105-125. doi:https://doi.org/10.1016/S0123-5923(08)70055-X

Hidalgo, L. (2013). La formación en emprendimiento como respuesta de la academia a las necesidades de la sociedad en el escenario ecuatoriano actual. RES NON VERBA, 4(4), 75-82.

doi:http://biblio.ecotec.edu.ec/revista/edicion4/LA\%20FORMACI\%C3\%93N\%20EN\% 20EMPRENDIMIENTO.pdf 
Holguín, A. (2013). La creación de redes: una estrategia para la enseñanza del emprendimiento en las IES. Praxis, 9, 99-107. doi:https://revistas.unimagdalena.edu.co/index.php/praxis/article/view/749

Juliá, J. (2012). Tribuna de opinión: emprendimiento y universidad. REVESCO Revista de $\begin{array}{lll}\text { Estudios } & \text { Cooperativos } & \text { 713, }\end{array}$ doi:http://dx.doi.org/10.5209/rev_REVE.2014.v113.43394

Kaplan, R., \& Norton , D. (1996). Using the Balanced Scorecard as a Strategic Management System. Harvard Business Review, 37-47. doi:https://d1wqtxts1xzle7.cloudfront.net/32320664/Kaplan_Norton_Balanced_Scoreca rd_-_3_articles.pdf?1384506447=\&response-content-

disposition=inline\%3B+filename\%3DPutting_the_Balanced_Scorecard_to_Work.pdf\& Expires $=1592171502 \&$ Signature $=$ XyDB-HziALiccjJ74-kUbhOi

Krauss, C. (2011). Actitudes emprendedoras de los estudiantes universitarios: El caso de la Universidad Católica del Uruguay. Dimensión Empresarial, 9(1), 28-40. doi:https://dialnet.unirioja.es/servlet/articulo?codigo=3797740

Landsdale, D., Abad, C., \& Vera, D. (2012). Impulsores Claves para Establecer el Ecosistema Dinámico de Emprendimiento en Ecuador. Polemika, 9(1), 18-25. doi:https://www.usfq.edu.ec/publicaciones/polemika/Documents/polemika009/polemika 009_007_articulo003.pdf

López, E., Rojas, L., \& Torres, A. (2001). Importancia estratégica del cuadro integral de mando en la gestión empresarial Balanced Scorecard. Ciencia e Ingeniería Neogranadina(11), 17-25. doi:https://doi.org/10.18359/rcin.1350

Maldonado, J. (2018). Metodología de la investigación social: Paradigmas: cuantitativo, sociocrítico, cualitativo, complementario. Bogotá: DGP Editores SAS.

Mc Coy, C., Martinez, M., \& Aguiar, P. (2019). Cancún, ciudad emergente: una propuesta de aplicación del modelo "Balance Scorecard" como método para evaluar la sostenibilidad y calidad de vida. El Periplo Sustentable, 241-273. doi:https://doi.org/10.36677/elperiplo.v0i37.9382

Méndez, J., \& Méndez, M. (2016). La influencia de la personalidad en los estilos del liderazgo. INNOVA Research Journal, 1(12), 52-58. doi:https://doi.org/10.33890/innova.v1.n12

Méndez, J., Ayala, H., \& Palacios, T. (2019). El Balanced Scorecarden el Desarrollo de los Negocios Familiares. INNOVA Research Journal, 4(3), 94-107. doi:https://doi.org/10.33890/innova.v4.n3.2019.963

Montoya, C. (2011). El Balanced Scorecard como herramienta de evaluacion en la gestión administrativa. Visión de Futuro, doi:http://revistacientifica.fce.unam.edu.ar/index.php?option=com_content\&view=articl e\&id=251\&Itemid $=61$

Morales, C., \& Pinilla, B. (2007). Balanced scorecard como herramienta de diagnóstico. Visión Gerencial, 6(1), 82-92. doi:https://www.redalyc.org/pdf/4655/465545875012.pdf

Muñoz, C. (2009). Cuadro de Mando Integral (Balanced Scorecard) para la gestión bibliotecaria: pautas para una aplicación. Investigacion Bibliotecológica, 23(48), 106-126. doi:http://dx.doi.org/10.22201/iibi.0187358xp.2009.48.16971

Neftalí, E. (12 de Noviembre de 2018). Balanced Scorecard Es La Principal Herramienta Metodológica Que Traduce La Estrategia en Un Conjunto de Medidas de La Actuación. Obtenido de https://es.scribd.com: https://es.scribd.com/document/338934613/BalancedScorecard-Es-La-Principal-Herramienta-Metodo 
Niven, P. (2003). El cuadro de mando integral paso a paso: maximizar la gestión y mantener los resultados. Barcelona: Gestión Pública.

Nuñez, L., \& Nuñez, M. (2016). Núñez, L., \& Núñez , M. (2016). Noción de emprendimiento para una formación escolar en competencia emprendedora/Notion for entrepreneurship in school entrepreneurial skills training. , 71, pp. 1069-1089, doi: 10.4185/RLCS-201. Revista Latina de Comunicación, 71, 1069-1089. doi:10.4185/RLCS-2016-1135

Peinado, J., Cerecedo, M., \& Jaramillo, D. (2016). El balanced scorecard, una herramienta de planeación estratégica en el Centro de Investigación e Innovación Tecnológica del Instituto Politécnico Nacional. UPIICSA. Investigación Interdisciplinaria, 32-50.

Pérez, P., \& García , L. (2014). La construcción de un cuadro de mando integral de tecnologías de la información en una empresa. Visión de Futuro, 18(2), 154-171. doi:https://revistacientifica.fce.unam.edu.ar/index.php?option=com_content\&view=artic le\&id=378:2014-07-15-18-33-48\&catid=157:2014-07-02-20-29-25

Quintero-Beltrán, L., \& Osorio-Morales, L. (2018). Balanced scorecard como herramienta para empresas en estado de crisis. Revista CEA, 4(8), 75-94. doi:https://doi.org/10.22430/24223182.1049

Rigby, D., \& Bilodeau, B. (11 de mayo de 2011). Management tools and Trends 2011. Obtenido de Bain \& Company: http://www.bain.com/Images/BAIN_BRIEF_Management_Tools.pdf

Ruiz , C., García, F., \& Delgado, N. (2014). Condicionantes de la intención emprendedora en el alumnado universitario: un análisis desde la perspectiva de género. Revista de Estudios Empresariales(2),

81-96. doi:https://pdfs.semanticscholar.org/dd42/b4b9c198a664f287b6cdd13023351c092159.p df

Salvador, C. (2008). Impacto de la inteligencia emocional percibida en la autoeficiencia emprendedora. Boletín de Psicología, doi:https://dialnet.unirioja.es/servlet/articulo?codigo=2732147

Sánchez, J., Vélez , M., \& Araújo, P. (2016). Balanced Scorecard para emprendedores: desde el modelo canvas al cuadro de mando integral. Revista Facultad de Ciencias Económicas: Investigación y Reflexión, 24(1), 37-47. doi:https://doi.org/10.18359/

Sánchez, J., Ward, A., Hernández, B., \& Florez, J. (2017). Educación emprendedora: estado del $\begin{array}{llll}\text { arte. } & \text { Propositos } & \text { y } & \text { Representaciones, }\end{array}$ doi:http://dx.doi.org/10.20511/pyr2017.v5n2.190

Sepúlveda, C., \& Reina, W. (2016). Sostenibilidad de los emprendimientos: Un análisis de los factores determinantes. Revista Venezolana de Gerencia, 33-49. doi:http://dx.doi.org/10.37960/revista.v21i73.21055

Sparano, H. (2014). Emprendimiento en América Latina y su impacto en la gestión de proyectos. Dimensión Empresarial, 12(2), 95-106. doi:10.15665/rde.v12i2.281

Suárez, F., \& González, M. (2017). Análisis de la Aplicación del Balanced Scorecard como Herramienta de Diagnóstico Adaptada a Microempresas. Revista de Economía \& Administración, 99-110. doi:1 0.7819/rbgn.v15i46.1175

Thompson, E. (2009). Individual entrepreneurial intent: construct clarification and development of an internationally reliable metric. . Entrepreneurship Theory \& Practice, 33(3), 664669. doi:https://www.effectuation.org/wp-content/uploads/2017/06/Thompson-2009Entrepreneurship_Theory_and_Practice.pdf 
Vásquez, J., \& Carrillo, M. (2010). La importancia de construir indicadores de gestión en las instituciones de educación superior apoyándose en balanced scorecard. Latin American and Caribbean Conference for Engineering and Technology (LACCEI'2010), 1-8. doi:http://www.laccei.org/LACCEI2010-Peru/published/ACC110_Vasquez.pdf

Villaroel, C., Cabrales, F., Fernández , A., \& Godoy , I. (2017). Indicadores de Innovación y Emprendimiento Aplicados a la Triple Hélice en la Reción de Arica y Parinacota, Chile. Interciencia, $42(11)$, 719-726. doi:https://www.redalyc.org/pdf/339/Resumenes/Resumen_33953499003_1.pdf 